\title{
Identification of pivotal genes and pathways for spinal cord injury via bioinformatics analysis
}

\author{
ZONGHAO ZHU $^{1}$, QIANG SHEN ${ }^{2}$, LIANG ZHU ${ }^{2}$ and XIAOKANG WEI ${ }^{2}$ \\ ${ }^{1}$ Department of Orthopedics, Shanghai Tian You Hospital, Shanghai 200331; \\ ${ }^{2}$ Department of Orthopedics, Shanghai General Hospital, Shanghai 200080, P.R. China
}

Received April 9, 2016; Accepted March 16, 2017

DOI: $10.3892 / \mathrm{mmr} .2017 .7060$

\begin{abstract}
The present study aimed to identify key genes and pathways associated with spinal cord injury (SCI) and subsequently investigate possible therapeutic targets for the condition. The array data of GSE20907 was downloaded from the Gene Expression Omnibus database and 24 gene chips, including 3-day, 4-day, 1-week, 2-week and 1-month post-SCI together with control propriospinal neurons, were used for the analysis. The raw data was normalized and then the differentially expressed genes (DEGs) in the (A) 2-week post-SCI group vs. control group, (B) 1-month post-SCI group vs. control group, (C) 1-month and 2-week post-SCI group vs. control group, and (D) all post-SCI groups vs. all control groups, were analyzed with a limma package. Gene Ontology annotation and Kyoto Encyclopedia of Genes and Genomes pathway enrichment analyses for DEGs were performed. Cluster analysis was performed using ClusterOne plugins. All the DEGs identified were associated with immune and inflammatory responses. Signal transducer and activator of transcription 3 (STAT3), erb-B2 receptor tyrosine kinase 4 (ERBB4) and cytochrome B-245, $\alpha$ polypeptide $(C Y B A)$ were in the network diagrams of (A), (C) and (D), respectively. The enrichment analysis of DEGs identified in all samples demonstrated that the DEGs were also enriched in the chemokine signaling pathway (enriched in STAT3) and the high-affinity immunoglobulin E receptor (FceRI) signaling pathway [enriched in proto-oncogene, src family tyrosine kinase $(L Y N)$ ]. Immune and inflammatory responses serve significant roles in SCI. STAT3, ERBB 4 and CYBA may be key genes associated with SCI at certain stages. Furthermore, STAT3 and $L Y N$ may be involved in the development of SCI via the chemokine and FceRI signaling pathways, respectively.
\end{abstract}

Correspondence to: Dr Qiang Shen, Department of Orthopedics, Shanghai General Hospital, 85 Wu Jin Road, Shanghai 200080, P.R. China

E-mail: shqiangshen@126.com

Key words: spinal cord injury, differentially expressed genes, Kyoto Encyclopedia of Genes and Genomes pathway, protein-protein interaction network

\section{Introduction}

Spinal cord injury (SCI) is caused by incident trauma or stroke, infection, inflammation and several congenital disorders $(1,2)$. In the UK, $\sim 40,000$ people suffer from SCI with an annual incidence of 13 per million (3). There are $\sim 60,000$ new cases of SCI reported in China and 12,000 new cases in the USA every year $(4,5)$. SCI may cause significant mortality and morbidity (6). There is no effective therapy for SCI and it is prevalent in a number of different countries $(7,8)$. Therefore, it is necessary to identify an effective treatment for SCI, and identify specific genes or pathways that are associated with SCI and may be able to provide novel ways of treating this condition.

A number of achievements associated with molecular mechanism of SCI have been made in previous years. The pathobiology of SCI comprises a primary mechanical insult to the spinal cord and a delayed secondary injury; the secondary injury may instigate apoptotic and necrotic cell death mechanisms (2). Neuroinflammation is a significant component for secondary injury in SCI (9) and the expression of apoptotic genes is increased, whereas the expression of anti-apoptotic genes is reduced (10). Axonal regeneration and functional recovery following SCI may be promoted by the inhibition of ephrin type-A receptor 4 in wild-type mice (11). A temporary block of the interleukin- 6 signaling pathway modifies the inflammatory response following SCI and thus promotes the regeneration of the spinal cord (12). Jin et al (13) suggested that the oxidative phosphorylation and central nervous system disease signaling pathways, in addition to the cell cycle and immune system pathways, served significant roles in the progression of SCI. Lai et al (14) indicated that immune response, olfactory transduction and cell cycle pathways were associated with SCI. However, certain key genes and pathways of SCI at different stages have not been completely studied. Further investigation is necessary to elucidate these key genes and pathways to provide possible therapeutic targets for the treatment of SCI.

Previous studies with the microarray data of GSE20907 have indicated that oligodendrocyte transcription factor 1 , activating transcription factor 3 , jun proto-oncogene, vascular endothelial growth factor A, cell division cycle 20, CD74 molecule, major histocompatibility complex, class II invariant chain, bromodomain containing 2 and SMAD 
family member 1 are key genes associated with SCI, and that SCI is associated with cardiovascular disease and cancers (13-15). The present study identified the differentially expressed genes (DEGs) at different stages of SCI based on the microarray data of GSE20907. Subsequently, functional enrichment analyses for DEGs were performed. A protein-protein interaction (PPI) network was constructed. The present study aimed to identify certain other key genes and pathways in SCI at different stages and investigate potential therapeutic targets of the disease.

\section{Materials and methods}

Microarray data. The array data of GSE20907 was downloaded from the Gene Expression Omnibus database, which is based on the platform of GPL6247 (Affymetrix Rat Gene 1.0 ST Array; Affymetrix; Thermo Fisher Scientific, Inc., Waltham, MA, USA) and was deposited by Siebert et al (16). A total of 24 gene chips, comprising 3-day, 4-day, 1-week, 2-week and 1-month post-SCI together with control propriospinal neurons, were used for the analysis. The sample numbers of every time point are presented in Table I.

Data preprocessing. The raw expression data were normalized using the robust multiarray average algorithm in oligo (17). Following the removal of the internal standard probe, the boxplot was performed for the expression values of every chip prior to and following normalization.

DEGs analysis. The DEGs in (A) 2-week post-SCI group vs. control group, (B) 1-month post-SCI group vs. control group, (C) 1-month and 2-week post-SCI group vs. control group, and (D) all post-SCI group vs. all control group, were analyzed with a limma package (18); $\mid \log _{2} \mathrm{FC}$ (fold-change) $\mid \geq 0.5$ and false discovery rate $\leq 0.01$ were set as the threshold criteria. Venn diagrams were plotted for these four comparison groups by a Venn diagrams package (19), and the overlaps of DEGs were obtained. The heatmaps were obtained for the expression values of DEGs by Pheatmap function (20) and if the identified DEGs could be distinguished the corresponding group was verified.

Gene Ontology (GO) and pathway enrichment analyses. The database for annotation, visualization and integrated discovery (DAVID) is an integrated data-mining environment and is used for the analysis of gene lists (21). GO (geneontology.org) is a tool used to annotate genes by collecting a defined, structured and controlled vocabulary (22). The Kyoto Encyclopedia of Genes and Genomes (KEGG) is a database used to put associated gene sets into their respective pathways (23).

The DEGs in four comparison groups were submitted to DAVID. Subsequently, GO annotation and KEGG pathway enrichment analyses were performed. Gene counts $\geq 5$ and a $\mathrm{P} \leq 0.01$ were set as the threshold values.

PPI network analysis. The DEGs were imported into the BisoGenet plugin in Cytoscape (24), known interactions were searched with the Biomolecular Interaction Network Database (BIND) (25) and the network diagrams were plotted. At the same time, cluster analysis was performed using default
Table I. Sample numbers of every time point.

\begin{tabular}{lcc}
\hline Time point & Control & Post-SCI \\
\hline 3 days & 0 & 4 \\
4 days & 4 & 0 \\
1 week & 4 & 0 \\
2 weeks & 4 & 2 \\
1 month & 4 & 2 \\
\hline
\end{tabular}

SCI, spinal cord injury.

parameter of the ClusterOne plugin in Cytoscape (26) with $\mathrm{P}<0.01$.

\section{Results}

Data preprocessing and DEG screening. The boxplots of samples prior to and following normalization are presented in Fig. 1. Volcano plots for the groups are presented in Fig. 2. The results demonstrated that the expression values of each sample were close to the same following normalization.

A total of 148 DEGs (31 downregulated and 117 upregulated) were obtained with $\mid \log _{2} \mathrm{FCl} \geq 0.5$ in group (A). The volcano plot is presented in Fig. 2A. A heat map by clustering analysis is presented in Fig. 3; it indicated that SP110 nuclear body protein and olfactory receptor 1645 separated the 2 -week post-SCI group from the control group. The top five genes with maximum change are presented in Table II.

A total of 128 DEGs (24 downregulated and 104 upregulated) were obtained with $\mid \log _{2} \mathrm{FCl} \geq 0.5$ in group (B). The volcano plot is presented in Fig. 2B. It was identified from the results of clustering analysis that cystatin-like 1 and ENSRNOT00000052640 separated the control group from the post-SCI group (Fig. 4). The top five genes with maximum change were presented in Table II.

A total of 133 DEGs (56 downregulated and 77 upregulated) were obtained with $\mid \log _{2} \mathrm{FCl} \geq 0.5$ in group $(\mathrm{C})$. The volcano plot was presented in Fig. 2C. A heat map by clustering analysis is presented in Fig. 5 and it indicated that cell death-inducing DFFA-like effector B and LOC689961 divided the samples into two groups. The top five genes with maximum change are presented in Table II.

A total of 238 DEGs (11 downregulated and 227 upregulated) were obtained with $\log _{2} \mathrm{FCl} \geq 0.5$ in group (D). The volcano plot is presented in Fig. 2D. From the results of clustering analysis, it was identified that abhydrolase domain containing 1 and ENSRNOTO0000053282 divided the samples into two groups (Fig. 6). The top five genes with maximum change are presented in Table II.

The Venn diagrams of the above four sets of DEGs [(A), (B), (C) and (D)] are presented in Fig. 7.

GO and pathway enrichment analyses. A total of $7 \mathrm{GO}$ terms, 6 GO terms and 251 (9 KEGG and 242 GO) terms were obtained in DEGs of (A), (B) and (D), respectively following functional clustering analysis of the DEGs of the above four sets; there was no significant enrichment results in the DEGs of (C). 

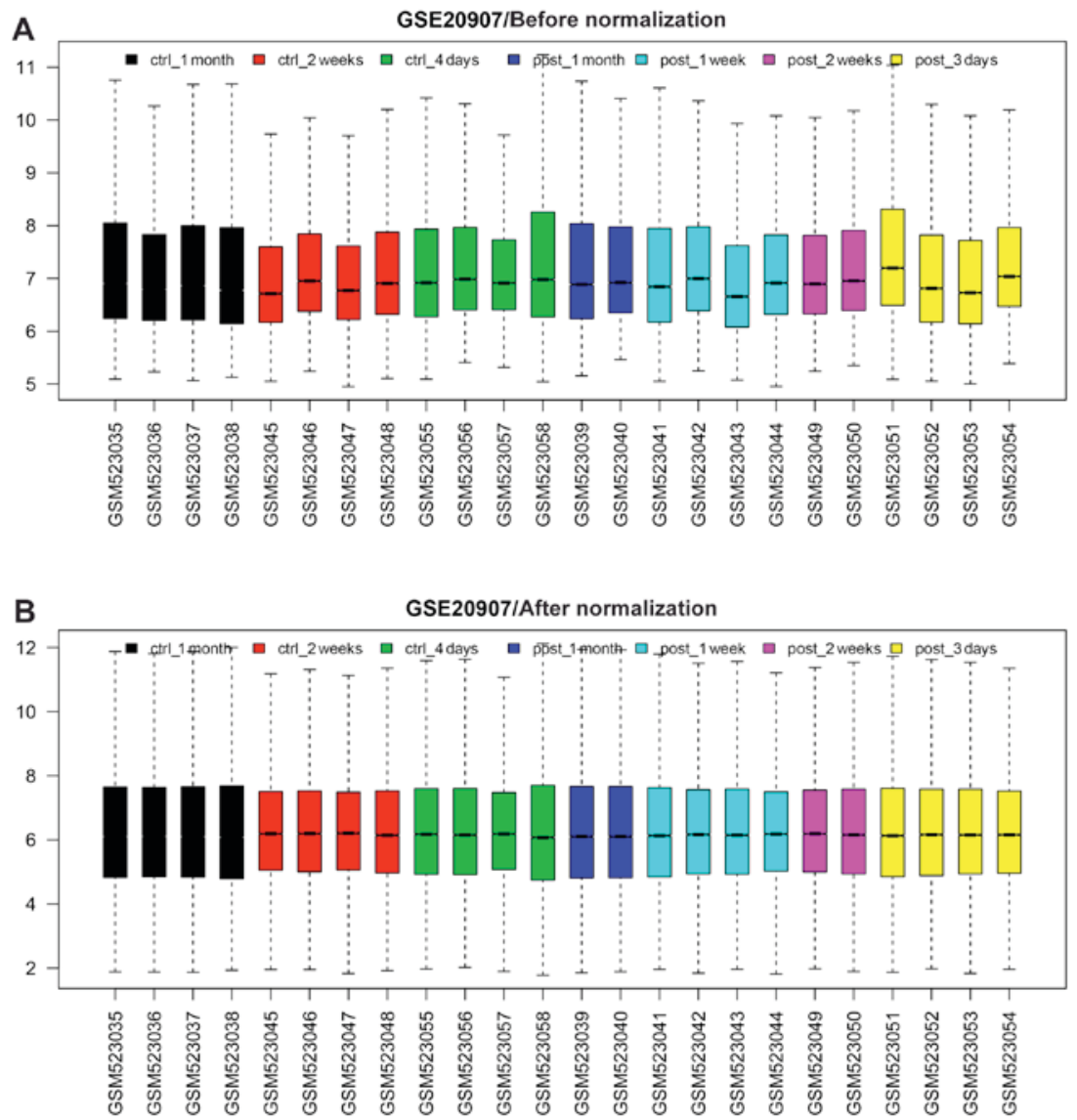

Figure 1. Boxplots of sample data (A) prior to and (B) following normalization. The lateral axis represents names of samples and the longitudinal axis represents expression levels. The horizontal line in the middle of post represents the expression levels of each sample. If the line of each sample was close to the same following normalization, then that indicated that all data were successfully normalized. Ctrl, control; post, post spinal cord injury.
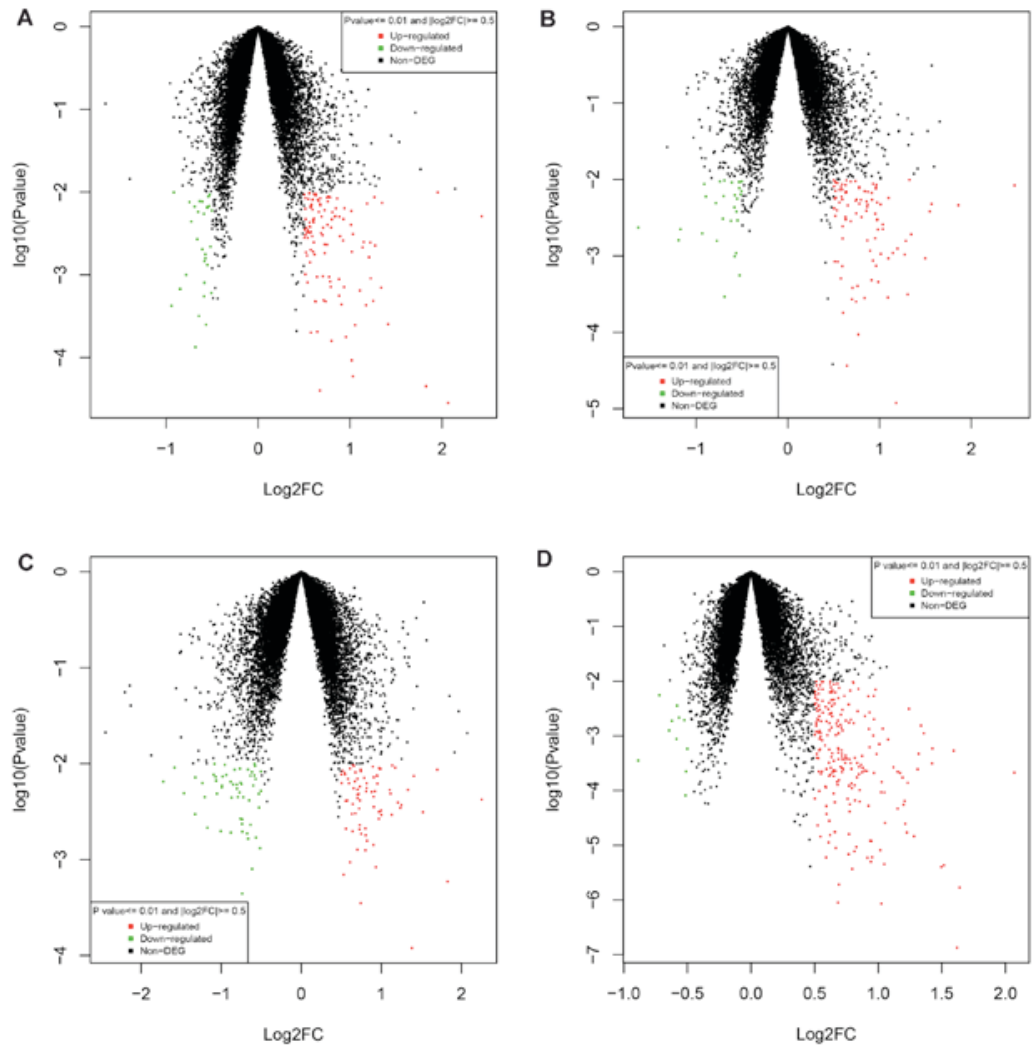

Figure 2. Volcano plot of (A) 2-week post-SCI group vs. control group, (B) 1-month post-SCI group vs. control group, (C) 1-month and 2-week post-SCI group vs. control group, and (D) all post-SCI groups vs. all control groups. SCI, spinal cord injury, FC, fold-change. 
Table II. Top five genes with maximum change in a 2-week time point, 1-month time point, 2-week and 1-month time point, and all samples respectively.

\begin{tabular}{|c|c|c|c|}
\hline Probe set ID & Log fold-change & P-value & Symbol \\
\hline \multicolumn{4}{|l|}{ 2-week } \\
\hline 10729312 & -0.94 & $4.21 \times 10^{-4}$ & ENSRNOT00000011249 \\
\hline 10847189 & -0.92 & $9.93 \times 10^{-3}$ & Olr697 \\
\hline 10910167 & -0.85 & $6.73 \times 10^{-4}$ & ENSRNOT00000020374 \\
\hline 10812399 & -0.78 & $1.00 \times 10^{-3}$ & Hapln1 \\
\hline 10846970 & -0.75 & $7.58 \times 10^{-3}$ & LOC100365431 \\
\hline 10761128 & 1.41 & $2.53 \times 10^{-4}$ & Hspb1 \\
\hline 10770710 & 1.83 & $4.50 \times 10^{-5}$ & Atf3 \\
\hline 10750524 & 1.95 & $9.93 \times 10^{-3}$ & Mx2 \\
\hline 10775624 & 2.06 & $2.84 \times 10^{-5}$ & ENSRNOT00000063555 \\
\hline 10753425 & 2.43 & $5.09 \times 10^{-3}$ & Mx1 \\
\hline \multicolumn{4}{|l|}{ 1-month } \\
\hline 10709450 & -1.63 & $2.35 \times 10^{-3}$ & Olr122 \\
\hline 10840577 & -1.19 & $1.60 \times 10^{-3}$ & Cstl1 \\
\hline 10707992 & -1.17 & $2.23 \times 10^{-3}$ & Klhl25 \\
\hline 10777123 & -0.93 & $1.97 \times 10^{-3}$ & ENSRNOT00000037316 \\
\hline 10904593 & -0.91 & $8.74 \times 10^{-3}$ & RGD1565410 \\
\hline 10938893 & 1.50 & $9.33 \times 10^{-4}$ & Mir325 \\
\hline 10735369 & 1.55 & $3.82 \times 10^{-3}$ & ENSRNOT00000052640 \\
\hline 10814301 & 1.57 & $4.79 \times 10^{-3}$ & ENSRNOT00000053252 \\
\hline 10887088 & 1.86 & $4.58 \times 10^{-3}$ & Mir544 \\
\hline 10775731 & 2.47 & $8.38 \times 10^{-3}$ & Cxcl13 \\
\hline \multicolumn{4}{|l|}{ 2-week and } \\
\hline \multicolumn{4}{|l|}{ 1-month } \\
\hline 10733056 & -1.72 & $6.50 \times 10^{-3}$ & Ifi47 \\
\hline 10775624 & -1.58 & $9.09 \times 10^{-3}$ & ENSRNOT00000063555 \\
\hline 10840577 & -1.46 & $4.92 \times 10^{-3}$ & Cstl1 \\
\hline 10909985 & -1.32 & $2.97 \times 10^{-3}$ & ENSRNOT00000053078 \\
\hline 10936645 & -1.32 & $7.15 \times 10^{-3}$ & RGD1563945 \\
\hline 10934666 & 1.41 & $7.43 \times 10^{-3}$ & ENSRNOT00000057449 \\
\hline 10769215 & 1.52 & $3.12 \times 10^{-3}$ & ENSRNOT00000059261 \\
\hline 10796258 & 1.70 & $8.65 \times 10^{-3}$ & ENSRNOT00000052594 \\
\hline 10878815 & 1.83 & $5.88 \times 10^{-4}$ & LOC690079 \\
\hline 10814301 & 2.25 & $4.23 \times 10^{-3}$ & ENSRNOT00000053252 \\
\hline \multicolumn{4}{|l|}{ All samples } \\
\hline 10888931 & -0.89 & $3.55 \times 10^{-4}$ & Abhd1 \\
\hline 10709450 & -0.72 & $5.53 \times 10^{-3}$ & Olr122 \\
\hline 10878210 & -0.64 & $1.25 \times 10^{-3}$ & Kank4 \\
\hline 10799241 & -0.62 & $1.87 \times 10^{-3}$ & Idi1 \\
\hline 10708471 & -0.59 & $8.79 \times 10^{-4}$ & Olr34 \\
\hline 10880731 & 1.52 & $4.31 \times 10^{-6}$ & $\mathrm{C} 1 \mathrm{qc}$ \\
\hline 10936482 & 1.59 & $5.38 \times 10^{-4}$ & Timp1 \\
\hline 10770710 & 1.62 & $1.34 \times 10^{-7}$ & Atf3 \\
\hline 10761128 & 1.64 & $1.69 \times 10^{-6}$ & Hspb1 \\
\hline 10775731 & 2.07 & $2.12 \times 10^{-4}$ & Cxcl13 \\
\hline
\end{tabular}

The functional enrichment analysis of group (A) demonstrated that the DEGs were primarily enriched in GO terms associated with wound and inflammatory responses, including
GO: 0009611-response to wounding and GO: 0002526-acute inflammatory response (Table III). The functional enrichment analysis of group (B) demonstrated that the DEGs 


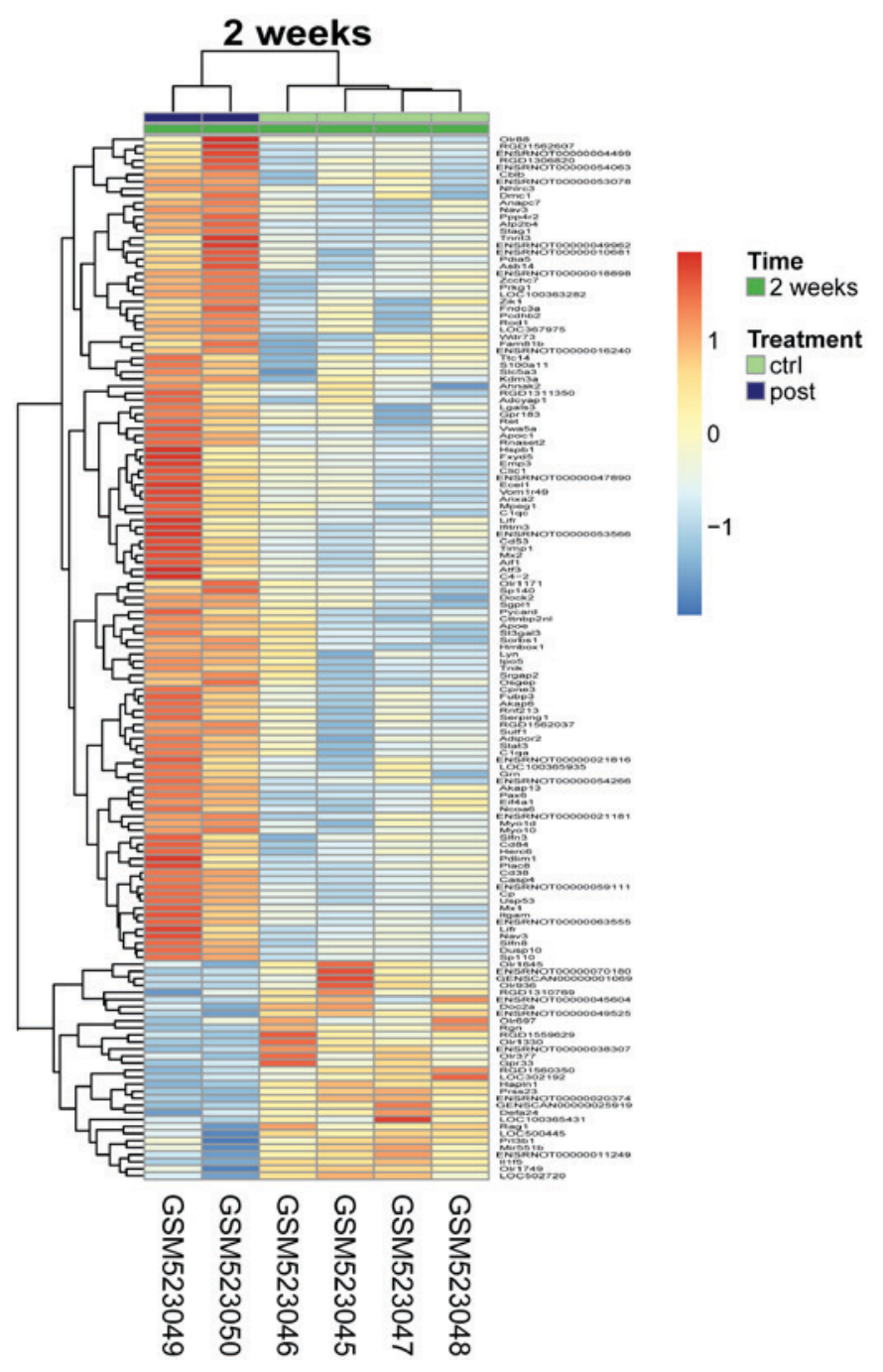

Figure 3. Heat maps of the differentially expressed genes in 2-week post-SCI group vs. control group. SCI, spinal cord injury; Ctrl, control; post, post-SCI.

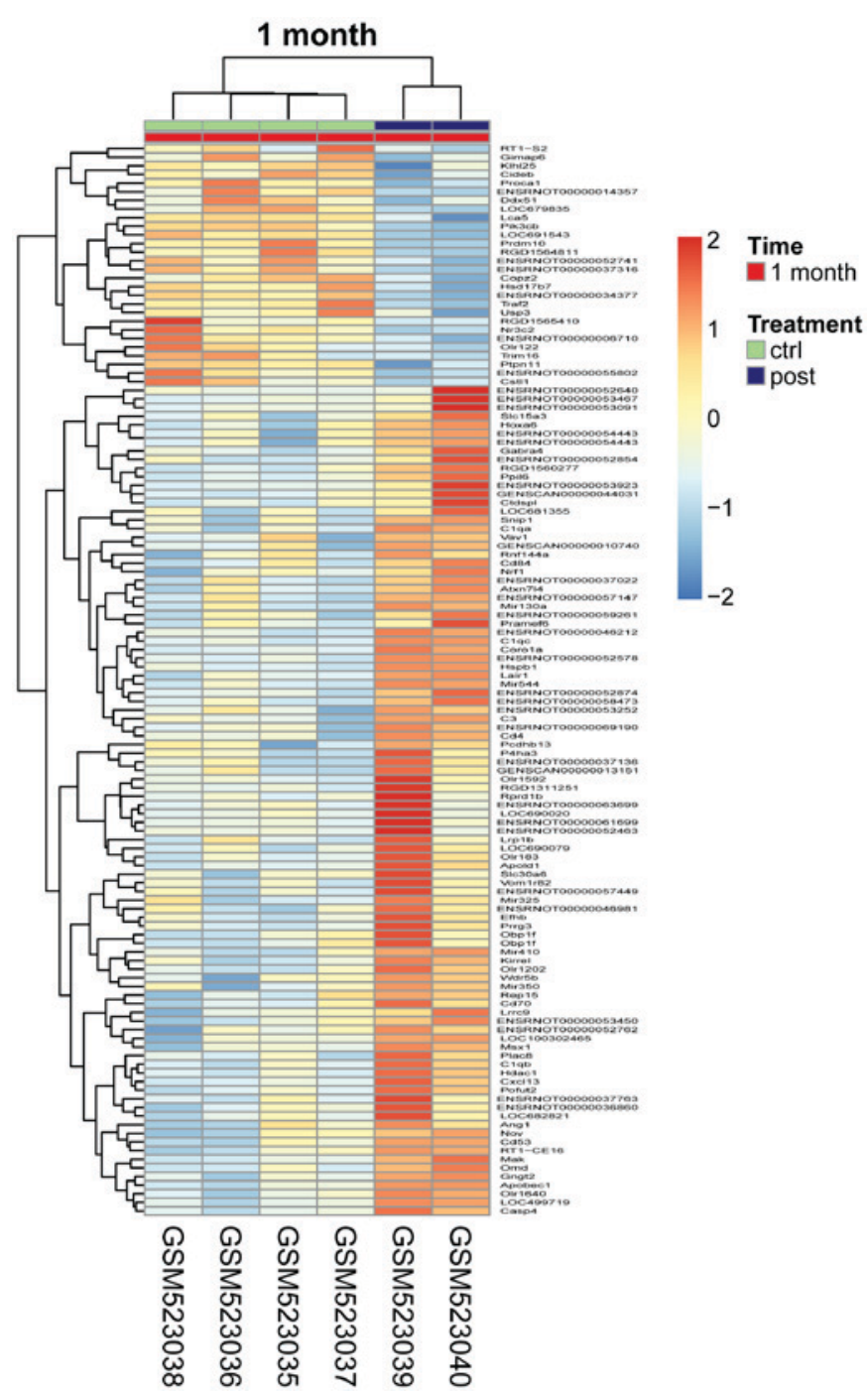

Figure 4. Heat maps of the differentially expressed genes in 1-month post-SCI group vs. control group. SCI, spinal cord injury; Ctrl, control; post, post-SCI. were primarily enriched in GO terms associated with the immune system and inflammatory response, including GO: 0006955-immune response and GO: 0006954-inflammatory response (Table III). The enrichment analysis of DEGs identified in all samples of the (D) group demonstrated that the DEGs were primarily enriched in the GO terms and pathways associated with the immune system and inflammatory response, including GO: 0006955-immune response and GO: 0006954-inflammatory response, in addition to chemokine signaling pathway and high-affinity immunoglobulin E receptor (FceRI) signaling pathway. Signal transducer and activator of transcription 3 (STAT3) and src family tyrosine kinase $(L Y N)$ are enriched in these two signaling pathways, respectively (Table III).

PPI network analysis. Following a BIND search, no known interaction among DEGs was identified in group (B). The network diagram of DEGs identified in group (A) is presented in Fig. 8A, and STAT3 and cbl proto-oncogene B, E3 ubiquitin protein ligase are in this diagram. Erb-B2 receptor tyrosine kinase $4(E R B B 4)$ was in the network diagram of DEGs identified in group (C; Fig. 8B). The network diagram of DEGs identified in group (D) is presented in Fig. 8C and cytochrome B-245, a polypeptide (CYBA), STAT3, protein tyrosine phosphatase, non-receptor type $6, \mathrm{~S} 100$ calcium binding protein A4 and Cluster of Differentiation 48 were in this diagram.

\section{Discussion}

In the present study, with the dataset of GSE20907, 148, 128, 133 and 238 DEGs were obtained in the 2-week post-SCI group vs. control group, 1-month post-SCI group vs. control group, 1-month and 2-week post-SCI group vs. control group, and all samples, respectively. The results demonstrated that the DEGs were mainly associated with the immune system and inflammatory response. STAT3, ERBB4, and $C Y B A$ were in the network diagram of 2-week post-SCI group vs. control group, 1-month and 2-week post-SCI group vs. control group, and all samples, respectively. The enrichment analysis of DEGs identified in all samples demonstrated that the DEGs were also enriched in the chemokine and FceRI signaling pathways, and that STAT3 and $L Y N$ are enriched in these two signaling pathways, respectively. 


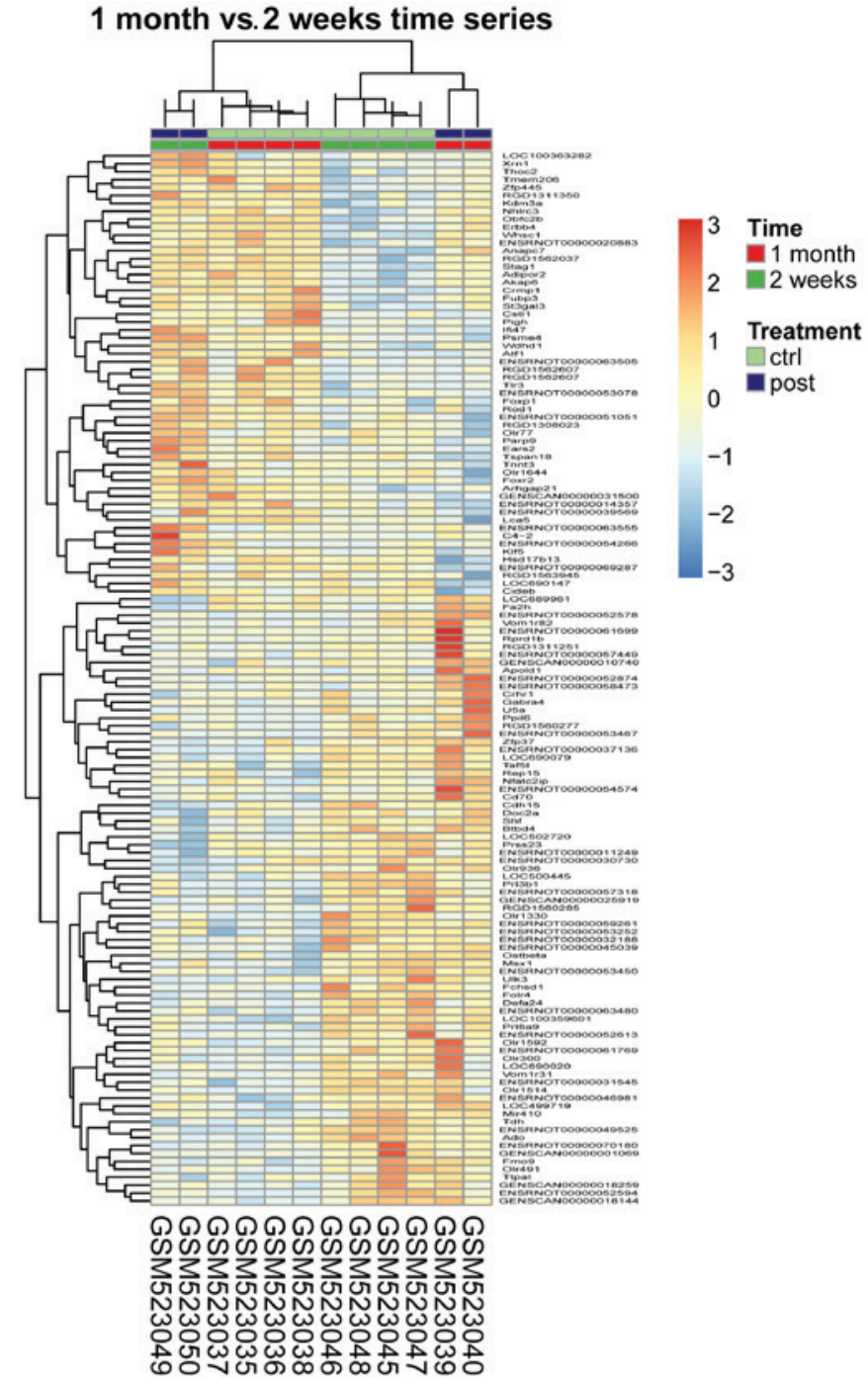

Figure 5. Heat maps of the differentially expressed genes in 1-month and 2-week post-SCI group vs. control group. SCI, spinal cord injury; Ctrl, control; post, post-SCI.

SCI may initiate immune responses by producing cytokines and chemokines, and by infiltration of peripheral leucocytes, and thus SCI-induced inflammation may cause a further deterioration in certain functions (27). Furthermore, it is indicated that inflammation serves a part in neuroprotection, axonal regeneration and functional recovery following SCI (28). Nuclear factor kappa-light-chain-enhancer of activated $\mathrm{B}$ cells $(\mathrm{NF}-\kappa \mathrm{B})$ is a pivotal regulator of inflammation and the suppression of astroglical $N F-\kappa B$ is able to reduce inflammation following SCI (29). Certain other studies also identify the important parts of the immune system and inflammatory response following SCI (30-32). In the present study, the DEGs were primarily associated with immune system and inflammatory response. The results therefore were in accord with previous studies and suggested that the immune system and inflammatory response served significant roles in the development of SCI.

In the present study, $E R B B 4$ and $C Y B A$ were in the network diagram of 1-month and 2-week post-SCI group vs. control group, and all samples, respectively. Lindholm et al (33) demonstrated that in the vicinity of the lesion, a number of scattered cells expressed $E r b B$ mRNA following SCI. Neuregulin1/ErbB signaling may be involved in the development of SCI (34). Nakano et al (35) indicated that vestibular and immune defects may be caused by the mutation of the $C Y B A$ gene encoding $\mathrm{p} 22^{\text {phox }}$. As stated above, the immune system serves an important role in the progression of SCI and thus, $C Y B A$ is associated with the progression of SCI via the immune system. Thus, the results of the present study were consistent with previous studies, and suggested that ERBB4 and $C Y B A$ may be key genes associated with $\mathrm{SCI}$ at a certain stage of the condition.

In the present study, the enrichment analysis of DEGs identified in all samples demonstrated that the DEGs were also enriched in the chemokine signaling pathway and that STAT3 is enriched in this signaling pathway. STAT3 was in the network diagram of the 2 -week post-SCI group vs. the control group. Chemokines may attract stem cells to the injured site and promote their survival following SCI (36). Taylor et al (37) suggested that cerebrospinal fluid cytokines and chemokines are dysregulated following acute thoracolumbar SCI in dogs. Chemokine-ligands/receptors are key mediator of secondary injury response following SCI (38) and STAT3, a transcription factor, is activated by tyrosine phosphorylation in response to epidermal growth factor and interleukin-6 (39). STAT3 is able to inhibit the spread of inflammation following SCI by regulating certain aspects of reactive astrogliosis, particularly the upregulation of glial scar formation and astrocyte hypertrophy, and the scar-forming astrocytes (40). Therefore, STAT3 may be a key gene associated with SCI and may be involved in the development of SCI via a chemokine signaling pathway.

$L Y N$, a non-receptor src-like tyrosine kinase, is a significant signaling mediator and is able to regulate apoptosis, differentiation and migration (41). Certain studies have suggested that $L Y N$ is involved in the process of oligodendrocyte differentiation $(42,43)$ and serves a vital role in myelination in the brain (44). The number of newly-formed oligodendrocytes and the branches of the myelin membrane are decreased in FYN proto-oncogene Src family tyrosine kinase-deficient mice (45). Certain studies have suggested that the death of oligodendrocytes may cause spinal cord dysfunction and that minocycline treatment may reduce delayed oligodendrocyte death following SCI $(46,47)$. In the present study, the enrichment analysis of DEGs identified in all samples demonstrated that the DEGs were also enriched in the FceRI signaling pathway and that $L Y N$ is enriched in this pathway. Although the roles of the FceRI signaling pathway have not been fully elucidated in SCI, it is hypothesized that $L Y N$ may be involved in the development of SCI via the FceRI signaling pathway.

In conclusion, the immune system and inflammatory response may serve significant roles in the development of SCI. STAT3, ERBB4 and CYBA may be key genes associated with SCI at a certain stage of the disorder. Furthermore, STAT3 and LYN may be involved in the development of SCI, via the chemokine and FceRI signaling pathways, respectively. However, as the sample size of the present study was small and no experimental verification was performed, further studies on the mechanisms of SCI development are required. 


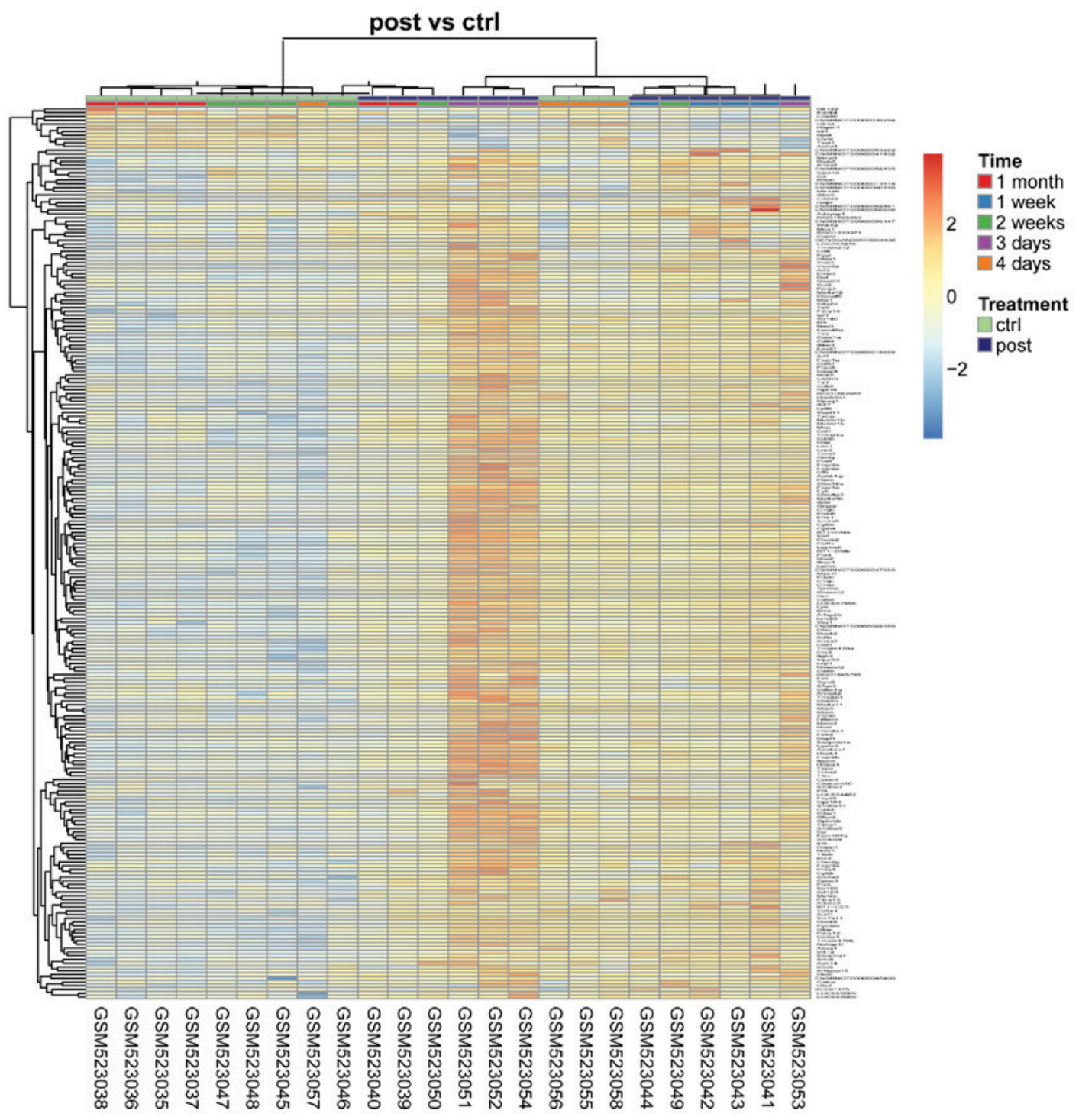

Figure 6. Heat maps of the differentially expressed genes in all post-SCI groups vs. all control groups. SCI, spinal cord injury; Ctrl, control; post, post-SCI.

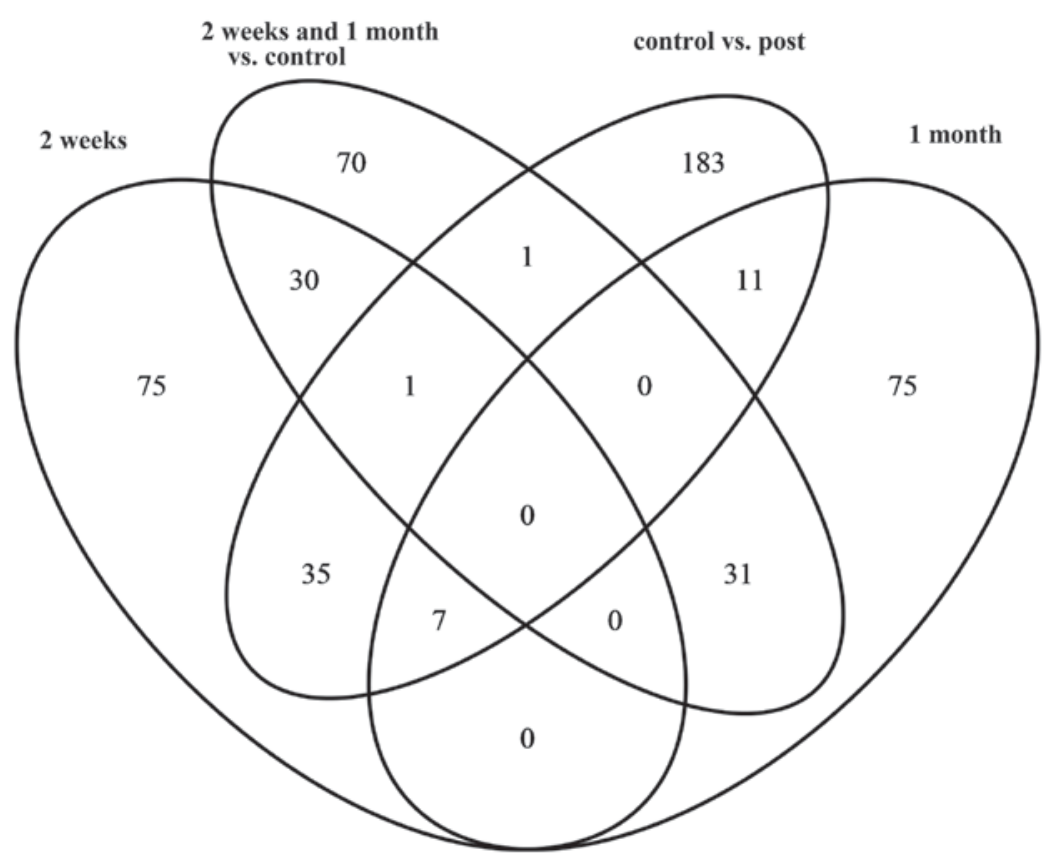

Figure 7. Venn diagrams of four sets of differentially expressed genes. Post, post-spinal cord injury. 
Table III. GO and KEGG pathway enrichment analysis for differentially expressed genes.

\begin{tabular}{|c|c|c|c|}
\hline Term & Description & Counts & P-value \\
\hline \multicolumn{4}{|l|}{ 2-week } \\
\hline GO:0009611 & Response to wounding & 10 & $1.72 \times 10^{-3}$ \\
\hline GO:0002526 & Acute inflammatory response & 5 & $2.82 \times 10^{-3}$ \\
\hline GO:0006954 & Inflammatory response & 7 & $2.92 \times 10^{-3}$ \\
\hline GO:0006952 & Defense response & 9 & $4.42 \times 10^{-3}$ \\
\hline GO:0001775 & Cell activation & 7 & $5.00 \times 10^{-3}$ \\
\hline GO:0034097 & Response to cytokine stimulus & 5 & $6.15 \times 10^{-3}$ \\
\hline GO:0051336 & Regulation of hydrolase activity & 7 & $7.39 \times 10^{-3}$ \\
\hline \multicolumn{4}{|l|}{ 1-month } \\
\hline GO:0006955 & Immune response & 11 & $2.16 \times 10^{-6}$ \\
\hline GO:0002684 & Positive regulation of immune system process & 8 & $2.13 \times 10^{-5}$ \\
\hline GO:0050778 & Positive regulation of immune response & 6 & $1.69 \times 10^{-4}$ \\
\hline GO:0002252 & Immune effector process & 5 & $1.04 \times 10^{-3}$ \\
\hline GO:0048584 & Positive regulation of response to stimulus & 6 & $1.63 \times 10^{-3}$ \\
\hline GO:0006954 & Inflammatory response & 5 & $8.46 \times 10^{-3}$ \\
\hline \multicolumn{4}{|l|}{ All samples } \\
\hline GO:0006955 & Immune response & 40 & $1.84 \times 10^{-25}$ \\
\hline GO:0006952 & Defense response & 37 & $8.08 \times 10^{-23}$ \\
\hline GO:0001775 & Cell activation & 28 & $1.51 \times 10^{-19}$ \\
\hline GO:0009611 & Response to wounding & 34 & $6.34 \times 10^{-19}$ \\
\hline GO:0006954 & Inflammatory response & 25 & $2.35 \times 10^{-17}$ \\
\hline KEGG: rno04650: & Natural killer cell mediated cytotoxicity & 13 & $3.22 \times 10^{-8}$ \\
\hline KEGG: rno05322: & Systemic lupus erythematosus & 9 & $6.82 \times 10^{-5}$ \\
\hline KEGG: rno04610: & Complement and coagulation cascades & 8 & $9.40 \times 10^{-5}$ \\
\hline KEGG: rno04662: & B cell receptor signaling pathway & 8 & $1.46 \times 10^{-4}$ \\
\hline KEGG: rno04060: & Cytokine-cytokine receptor interaction & 12 & $2.11 \times 10^{-4}$ \\
\hline
\end{tabular}

GO, Gene Ontology; KEGG, Kyoto Encyclopedia of Genes and Genomes.

A

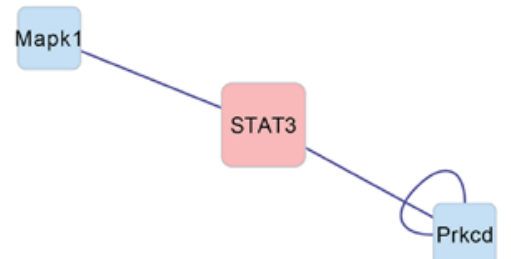

C

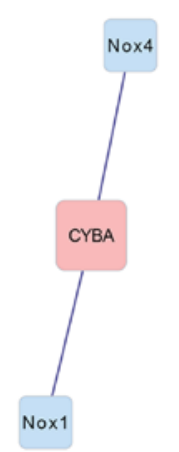

B
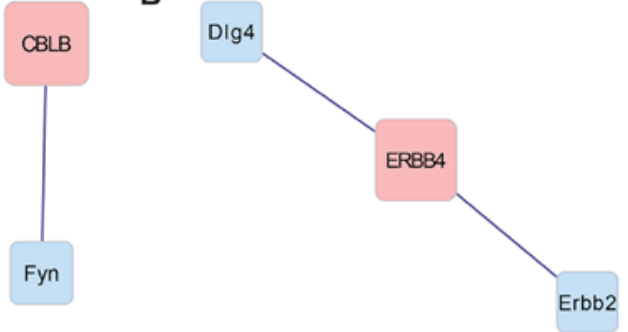

Erbb2

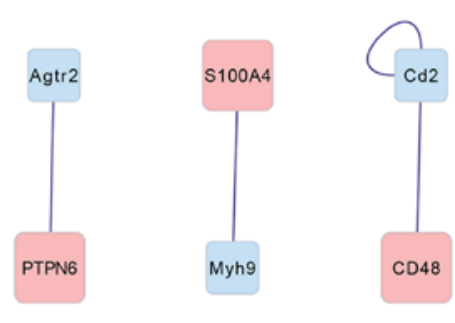

Figure 8. Network diagram of (A) 2-week time points, (B) 2-week time and 1-month time series analysis and (C) the comparison between all control groups and all post-SCI groups. 


\section{References}

1. Mueller JB: Spinal cord injury. In: Preanesthetic Assessment 3 Frost EAM (ed). Birkhäuser, Boston, USA: pp11-13, 2012.

2. Baptiste DC and Fehlings MG: Pharmacological approaches to repair the injured spinal cord. J Neurotrauma 23: 318-334, 2006.

3. Winter B, Pattani H and Temple E: Spinal cord injury. Anaesth Intensive Care 15: 424-427, 2014.

4. Qiu J: China spinal cord injury network: Changes from within. Lancet Neurol 8: 606-607, 2009.

5. National Spinal Cord Injury Statistical Center: Spinal cord injury facts and figures at a glance. J Spinal Cord Med 36: 1-2, 2013.

6. Shah RR and Tisherman SA: Spinal cord injury. In: Imaging the ICU Patient. Springer, pp377-380, 2014.

7. Sekhon LH and Fehlings MG: Epidemiology, demographics, and pathophysiology of acute spinal cord injury. Spine (Phila Pa 1976) 26 (24 Suppl): S2-S12, 2001.

8. Wyndaele M and Wyndaele JJ: Incidence, prevalence and epidemiology of spinal cord injury: What learns a worldwide literature survey? Spinal cord 44: 523-529, 2006.

9. Munro KM, Perreau VM and Turnley AM: Differential gene expression in the EphA4 knockout spinal cord and analysis of the inflammatory response following spinal cord injury. PLoS One 7: e37635, 2012.

10. Kotipatruni RR, Dasari VR, Veeravalli KK, Dinh DH, Fassett D and Rao JS: p53-and Bax-mediated apoptosis in injured rat spinal cord. Neurochem Res 36: 2063-2074, 2011.

11. Goldshmit Y, Spanevello MD, Tajouri S, Li L, Rogers F, Pearse M, Galea M, Bartlett PF, Boyd AW and Turnley AM: EphA4 blockers promote axonal regeneration and functional recovery following spinal cord injury in mice. PLoS One 6: e24636, 2011.

12. Guerrero AR, Uchida K, Nakajima H, Watanabe S, Nakamura M, Johnson WE and Baba H: Blockade of interleukin-6 signaling inhibits the classic pathway and promotes an alternative pathway of macrophage activation after spinal cord injury in mice. J Neuroinflammation 9: 40, 2012.

13. Jin L, Wu Z, Xu W, Hu X, Zhang J, Xue Z and Cheng L: Identifying gene expression profile of spinal cord injury in rat by bioinformatics strategy. Mol Biol Rep 41: 3169-3177, 2014.

14. Lai J, He X, Wang F, Tan JM, Wang JX, Xing SM, Shen LB, Fang LQ, Yang P and Tan JM: Gene expression signature analysis and Protein-Protein interaction network construction of Spinal Cord Injury. Eur Rev Med Pharmacol Sci 17: 2941-2948, 2013.

15. Wang W, Liu R, Xu Z, Niu X, Mao Z, Meng Q and Cao X Further insight into molecular mechanism underlying thoracic spinal cord injury using bioinformatics methods. Mol Med Rep 12: 7851-7858, 2015.

16. Siebert JR, Middelton FA and Stelzner DJ: Intrinsic response of thoracic propriospinal neurons to axotomy. BMC Neurosci 11: 69, 2010.

17. Carvalho BS and Irizarry RA: A framework for oligonucleotide microarray preprocessing. Bioinformatics 26: 2363-2367, 2010.

18. Smyth GK: Limma: Linear models for microarray data. In: Bioinformatics and computational biology solutions using $\mathrm{R}$ and Bioconductor. Springer, pp397-420, 2005.

19. Chen H and Boutros PC: VennDiagram: A package for the generation of highly-customizable Venn and Euler diagrams in R. BMC Bioinformatics 12: 35, 2011.

20. Kolde R: Pheatmap: Pretty heatmaps. R package version 61, 2012 - https://CRAN.R-project.org/package=pheatmap. Accessed October 22, 2015.

21. Huang da W, Sherman BT and Lempicki RA: Systematic and integrative analysis of large gene lists using DAVID bioinformatics resources. Nat Protoc 4: 44-57, 2008.

22. Ashburner M, Ball CA, Blake JA, Botstein D, Butler H, Cherry JM, Davis AP, Dolinski K, Dwight SS, Eppig JT, et al: Gene Ontology: Tool for the unification of biology. The Gene Ontology Consortiumm. Nat Genet 25: 25-29, 2000.

23. Altermann E and Klaenhammer TR: PathwayVoyager: Pathway mapping using the Kyoto Encyclopedia of Genes and Genomes (KEGG) database. BMC Genom 6: 60, 2005.

24. Martin A, Ochagavia ME, Rabasa LC, Miranda J, Fernandez-de-Cossio J and Bringas R: BisoGenet: A new tool for gene network building, visualization and analysis. BMC Bioinformatics 11: 91, 2010.

25. Bader GD, Betel D and Hogue CW: BIND: The biomolecular interaction network database. Nucleic Acids Res 31: 248-250, 2003.

26. Nepusz T, Yu H and Paccanaro A: Detecting overlapping protein complexes in protein-protein interaction networks. Nat Methods 9: 471-472, 2012.
27. Hausmann ON: Post-traumatic inflammation following spinal cord injury. Spinal cord 41: 369-378, 2003.

28. Donnelly DJ and Popovich PG: Inflammation and its role in neuroprotection, axonal regeneration and functional recovery after spinal cord injury. Exp Neurol 209: 378-388, 2008.

29. Brambilla R, Bracchi-Ricard V, Hu WH, Frydel B, Bramwell A, Karmally S, Green EJ and Bethea JR: Inhibition of astroglial nuclear factor kappaB reduces inflammation and improves functional recovery after spinal cord injury. J Exp Med 202: 145-156, 2005.

30. Esposito E, Bruscoli S, Mazzon E, Paterniti I, Coppo M, Velardi E, Cuzzocrea S and Riccardi C: Glucocorticoid-induced leucine zipper (GILZ) over-expression in T lymphocytes inhibits inflammation and tissue damage in spinal cord injury. Neurotherapeutics 9: 210-225, 2012.

31. Zhang Y, Guan Z, Reader B, Shawler T, Mandrekar-Colucci S, Huang K, Weil Z, Bratasz A, Wells J, Powell ND, et al: Autonomic dysreflexia causes chronic immune suppression after spinal cord injury. J Neurosci 33: 12970-12981, 2013.

32. de Rivero Vaccari JP, Minkiewicz J, Wang X, De Rivero Vaccari JC, German R, Marcillo AE, Dietrich WD and Keane RW: Astrogliosis involves activation of retinoic acidinducible gene-like signaling in the innate immune response after spinal cord injury. Glia 60: 414-421, 2012.

33. Lindholm T, Cullheim S, Deckner M, Carlstedt T and Risling M: Expression of neuregulin and ErbB3 and ErbB4 after a traumatic lesion in the ventral funiculus of the spinal cord and in the intact primary olfactory system. Exp Brain Res 142: 81-90, 2002.

34. Tao F, Li Q, Liu S, Wu H, Skinner J, Hurtado A, Belegu V, Furmanski O, Yang Y, McDonald JW and Johns RA: Role of Neuregulin-1/ErbB signaling in stem cell therapy for spinal cord injury-induced chronic neuropathic pain. Stem Cells 31: 83-91, 2013.

35. Nakano Y, Longo-Guess CM, Bergstrom DE, Nauseef WM, Jones SM and Bánfi B: Mutation of the Cyba gene encoding p22phox causes vestibular and immune defects in mice. J Clin Invest 118: 1176-1185, 2008.

36. Hassanshahi G, Amin M, Shunmugavel A, Vazirinejad R, Vakilian A, Sanji M, Shamsizadeh A, RafatPanah H, Poor NM, Moosavi SR and Taheri S: Temporal expression profile of CXC chemokines in serum of patients with spinal cord injury. Neurochem Int 63: 363-367, 2013.

37. Taylor AR, Welsh CJ, Young C, Spoor E, Kerwin SC, Griffin JF, Levine GJ, Cohen ND and Levine JM: Cerebrospinal fluid inflammatory cytokines and chemokines in naturally occurring canine spinal cord injury. J Neurotrauma 31: 1561-1569, 2014.

38. Knerlich-Lukoschus F and Held-Feindt J: Chemokine-ligands/ receptors: Multiplayers in traumatic spinal cord injury. Mediators Inflamm 2015: 486758, 2015.

39. Zhong Z, Wen Z and Darnell JE jr: Stat3: A STAT family member activated by tyrosine phosphorylation in response to epidermal growth factor and interleukin-6. Science 264: 95-98, 1994.

40. Herrmann JE, Imura T, Song B, Qi J, Ao Y, Nguyen TK, Korsak RA, Takeda K, Akira S and Sofroniew MV: STAT3 is a critical regulator of astrogliosis and scar formation after spinal cord injury. J Neurosci 28: 7231-7243, 2008.

41. Ingley E: Functions of the Lyn tyrosine kinase in health and disease. Cell Commun Signal 10: 21, 2012.

42. Sperber BR, Boyle-Walsh EA, Engleka MJ, Gadue P, Peterson AC, Stein PL, Scherer SS and McMorris FA: A unique role for Fyn in CNS myelination. J Neurosci 21: 2039-2047, 2001.

43. Goto J, Tezuka T, Nakazawa T, Sagara $\mathrm{H}$ and Yamamoto $\mathrm{T}$ : Loss of Fyn tyrosine kinase on the C57BL/6 genetic background causes hydrocephalus with defects in oligodendrocyte development. Mol Cell Neurosci 38: 203-212, 2008.

44. Hossain S, Fragoso G, Mushynski WE and Almazan G: Regulation of peripheral myelination by Src-like kinases. Exp Neurol 226: 47-57, 2010.

45. Sperber BR and McMorris FA: Fyn tyrosine kinase regulates oligodendroglial cell development but is not required for morphological differentiation of oligodendrocytes. J Neurosci Res 63: 303-312, 2001.

46. Stirling DP, Khodarahmi K, Liu J, McPhail LT, McBride CB, Steeves JD, Ramer MS and Tetzlaff W: Minocycline treatment reduces delayed oligodendrocyte death, attenuates axonal dieback, and improves functional outcome after spinal cord injury. J Neurosci 24: 2182-2190, 2004.

47. Beattie MS, Farooqui AA and Bresnahan JC: Review of current evidence for apoptosis after spinal cord injury. J Neurotrauma 17: 915-925, 2000. 